\title{
Growth and thermal stability of TiN/ZrAIN: Effect of internal interfaces
}

Kumar Yalamanchili, Fei Wang, Hisham Aboulfadl, Jeni Barrirero, Lina Rogström, F. Mücklich, Ferenc Tasnadi, Emilio Jimenez Pique, Magnus Odén and Naureen Ghafoor.

\begin{abstract}
Wear resistant hard films comprised of cubic (c) transition metal nitride (TMN) and metastable $c$-AlN with coherent interfaces have a confined operating envelope governed by the thermal stability of present metastable phases. However, films grown with equilibrium phases (c-TMN and wurtzite $(w)$ AlN) but with semi-coherent interfaces potentially have, higher thermal stability. We demonstrate this concept for a model multilayer system consisting TiN and ZrAlN layers where the high growth temperature leads to the formation of ZrN- and AlN- rich domains during growth. The multilayer architecture and growth temperature are varied to tune the crystal and interface structure of AlN. At higher growth temperature, when the adatoms have sufficient surface mobility, the interface energy minimization leads to formation of semi-coherent interfaces between $w$-AlN and $c$-TMN during the growth. The combined effects of relatively higher thermodynamic stability of semicoherent $w$-AlN/c-TMN interface structure and confinement of $w$-AlN to nm-sized domains by the immiscible $c$-TMN, leads to high thermal stability of the multilayers with a stable hardness of $34 \pm 1.5 \mathrm{GPa}$ after annealing to $1150{ }^{\circ} \mathrm{C}$ for $2 \mathrm{~h}$. Ab initio calculations predict higher thermodynamic stability of semi-coherent interfaces between c-TMN and $w$-AlN than isostructural coherent interface between c-TMN and $c$-AlN.
\end{abstract}




\section{Introduction}

Material development for wear resistant thin films with high hardness at elevated temperature is a long- standing technological challenge. The current workhorse material for tool coatings is TiAlN with $50-67$ at. $\%$ of $\mathrm{Al}$ on the metallic sublattice. TiAlN films exhibit hardness enhancement at annealing temperatures between 700 and $900{ }^{\circ} \mathrm{C}$ due to spinodal decomposition of supersaturated cubic (c)-TiAlN in to $c$-TiN and metastable $c$-AlN [1-3]. The iso-structural domains form coherent interfaces, however the lattice coherency breakdown above $900{ }^{\circ} \mathrm{C}$ when $c$-AlN transforms to the thermodynamically stable wurtzite (w) phase [2]. The resulting incoherent interfaces cause hardness drop and thus limit the working envelope of the film [2]. Several approaches, such as multicomponent alloying (Chen et al. 2011; Rachbauer et al. 2012), multilayering [8], and interface coherency strain tuning [9] have been developed to enhance the stability of the metastable $c$-AlN with respect to $w$ AlN. Nevertheless, there is a temperature limit around $1000^{\circ} \mathrm{C}$, above which the metastable $c$ AlN assume its thermodynamically stable wurtzite structure [8]. In addition, a large volume expansion associated with the transformation leads to structural instability of the film and limits the application temperature envelope [10].

In this article, we investigate an alternative design route for improving the thermal stability of AlN containing transition metal nitride (TMN) thin films. In place of the above mentioned approaches for stabilizing metastable $c$-AlN we propose an alternative structural archetype consisting $c$-TMN and thermodynamically stable $w$-AlN with semi-coherent interfaces. The concept originates from recent experimental studies showing films consisting of nonisostructural but semi-coherent internal interfaces that exhibit hardness values similar to films with iso-structural coherent interfaces between $c$-TiN and $c$-AlN [11-14]. It exposes the fact that even though $w$-AlN has a lower shear resistance [15], coatings containing $w$-AlN could be strengthened by growing them such that semi-coherent interfaces are formed. The current 
knowledge of semi-coherent growth of $w$-AlN is, however, limited $[12,13,16]$. Their thermal stability is yet to be studied, which we address in this article using TiN/ZrAlN as a model system.

ZrAlN is a strongly immiscible alloy with a maximum enthalpy of mixing for the composition $\mathrm{Zr}_{0.4} \mathrm{Al}_{0.6} \mathrm{~N}[17,18]$, the one chosen for this study. ZrAlN undergoes segregation to its binaries $\mathrm{ZrN}$ and AlN during high temperature growth [11]. By adapting a multilayer structure it is shown that the crystal structure of AlN can be tuned between the cubic and wurtzite phases by varying the layer thickness [19-21]. Here we combine these phenomena to explore how the interface structure between $w$-AlN and either $c$-TiN or $c-\mathrm{ZrN}$ can be tuned by sandwiching the compositionally modulated structure of ZrAlN between the TiN layers. Note that the term "interface" is here used to describe layer interfaces as well as boundaries between chemically segregated domains and collectively all interfaces are called "internal interfaces".

The multilayers of TiN/ZrAlN are grown by reactive magnetron sputter deposition where the thickness of ZrAlN layer and the growth temperature is varied to switch between isostructural $(c-\mathrm{TMN} / c-\mathrm{AlN})$ and hetrostructural $(c-\mathrm{TMN} / w-\mathrm{AlN}) \quad$ coherent/semi-coherent interfaces. We have compared the relative thermodynamic stability of iso-structural and nonisostructural coherent interface structures by first principle calculations and combined it with elevated temperature annealing experiments. The results provide insight in to the interface crystallography and chemistry requirements to obtain a thermally stable nanocomposite. In addition, the effect of internal interface on the mechanical properties is investigated by performing nano hardness tests on as deposited and annealed films.

The study suggests a generic design route of enhanced thermal stability of the hard coatings by having semi-coherent $c$-TMN (111) /w-AlN (0001) interfaces as a structural archetype. Instead of improving the stability of metastable phases, the approach of designing thermally stable multiphase materials with tailored interface structures provides a design concept for 
materials to be used at elevated temperatures and with possibility to be extended to bulk-form materials.

\section{Experimental and calculation methods}

$\mathrm{TiN} / \mathrm{Zr}_{0.43} \mathrm{Al}_{0.57} \mathrm{~N}$ multilayer films were deposited on $\mathrm{MgO}(001)$ substrates in a high vacuum dual DC magnetron sputter deposition system with a background pressure of $2 \times 10^{-5} \mathrm{~Pa}$. Details of the deposition chamber can be found elsewhere [22]. A pure Ti and a pre-alloyed $\mathrm{Zr}_{0.4} \mathrm{Al}_{0.6}$ target were used. The discharge was obtained at $\mathrm{N}_{2}$ and $\mathrm{Ar}$ partial pressures of 0.06 and $0.5 \mathrm{~Pa}$, respectively. Applied powers of $\mathrm{P}_{\mathrm{Ti}}=200 \mathrm{~W}$ and $\mathrm{P}_{\mathrm{Zr} 0.4 \mathrm{Al} 0.6}=150 \mathrm{~W}$ resulted in deposition rates of $0.15(\mathrm{TiN})$ and $0.18\left(\mathrm{Zr}_{0.43} \mathrm{Al}_{0.57} \mathrm{~N}\right) \mathrm{nm} / \mathrm{s}$, respectively. The individual layers thicknesses (l) of TiN and $\mathrm{Zr}_{0.43} \mathrm{Al}_{0.57} \mathrm{~N}$ in the modulated structure were controlled by shutters in front of each target. The nominal thickness $l_{\text {TiN }}$ was kept constant at $15 \mathrm{~nm}$, whereas $l_{\text {ZrAlN }}$ was $2,5,10,15$, and $30 \mathrm{~nm}$ in a growth series of five films.

Monolithic films of $\mathrm{TiN}$ and $\mathrm{Zr}_{0.43} \mathrm{Al}_{0.57} \mathrm{~N}$ (with a $30 \mathrm{~nm}$ thick TiN buffer layer) were also deposited for reference. A total layer thickness of about $1 \mu \mathrm{m}$ was achieved for all samples to ensure reliable hardness measurements. All films were grown at a temperature of $\mathrm{T}_{\mathrm{s}}=700{ }^{\circ} \mathrm{C}$. An additional multilayer with $l_{\text {ZrAIN }}=15 \mathrm{~nm}$ was also deposited at $\mathrm{T}_{\mathrm{s}}=900{ }^{\circ} \mathrm{C}$ to map the favorable growth temperature for the $w$-AIN with semi-coherent interfaces. This film was further annealed at $1150{ }^{\circ} \mathrm{C}$ for 2 hours under controlled atmosphere of $95 \% \mathrm{~N}_{2}$ and $5 \% \mathrm{H}_{2}$ to probe thermal stability.

The composition of the monolithic films was determined by elastic recoil detection analysis (ERDA) using a $40 \mathrm{MeV} \mathrm{I}^{+}$beam. Structural changes were characterized by X-ray diffraction (XRD) with a Panalytical Empyrian diffractometer operated in Bragg-Brentano geometry using $\mathrm{Cu}-\mathrm{K}_{\alpha}$ radiation. Transmission electron microscopy (TEM) and scanning transmission electron microscopy (STEM) were performed using a FEI Tecnai G2 microscope operated at $200 \mathrm{kV}$, equipped with an energy- dispersive X-ray analysis spectrometer (EDX). For STEM 
analysis, a high angular annular dark field (HAADF) detector with a camera length of 160 mm was used. Cross section TEM (X-TEM) samples were prepared by conventional mechanical polishing followed by ion milling [14].

Atom probe tomography (APT) was used to obtain quantitative information regarding the interface chemistry. APT was performed on four films; $l_{\text {ZrAlN }} 2$ and $15 \mathrm{~nm}\left(\mathrm{~T}_{\mathrm{s}} 700^{\circ} \mathrm{C}\right), l_{\text {ZralN }}\left(\mathrm{T}_{\mathrm{s}}\right.$ $900^{\circ} \mathrm{C}$ ) in the as deposited state and after iso-thermal annealing. Atom probe specimen preparations were done in a dual-beam focused ion beam/SEM workstation implementing the in situ lift out technique [23]. A $200 \mathrm{~nm}$ thick electron beam Pt layer was deposited on the film surface to reduce Ga implantation during specimen preparation. The measurements were carried out using a LEAPTM $3000 X$ HR CAMECA $^{\text {TM }}$ system in laser pulsing mode $(532 \mathrm{~nm}$ wavelength) with repetition rates of $160 \mathrm{kHz}$, base temperatures of $40-50 \mathrm{~K}$, and a laser pulse energies of $0.4-0.5 \mathrm{~nJ}$. The data were reconstructed using the standard algorithm developed by

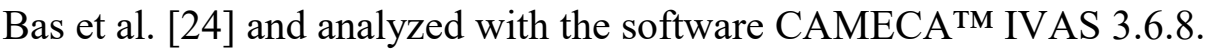

First principle calculations were performed to compare the relative thermodynamic stability between iso-structural and non iso-structural interfaces. Multilayers of TiN/AlN and ZrN/AlN were modelled with different interface structures. The total energies were calculated for the different interfaces with the smallest $(1 \times 1)$ in-plane periodicity. The interface models contain the same ratio of $\mathrm{TM} / \mathrm{N}$ atoms in each layer. These conditions results in different slab thicknesses for different multilayers. These thicknesses were varied until the relative energy differences between the different multilayers were consistent, which was achieved with slabs of $12 \mathrm{Ti}$ or $\mathrm{Zr}$ atoms and 24 Nitrogen atoms altogether. The level of accuracy is sufficient for comparative conclusions.

The total energy calculations were performed within the density functional theory (DFT) using the projector augmented wave (PAW) approach [25] implemented in the Vienna Ab 
initio Simulation Package (VASP) [26]. The Perdew-Burke-Ernzerhof generalized gradient functional (PBE-GGA) [27] was used to approximate the exchange and correlation functional and the plane-wave energy cutoff value of $500 \mathrm{eV}$ was used. The reciprocal space integration was performed within the Monkhorst-Pack scheme [28] using a k-mesh of $5 \times 5 \times 1$. To determine the thermodynamic equilibrium configuration of the multilayers, the in-plane lattice parameter, the c/a ratio and all the atomic coordinates were relaxed.

Hardness and Young's modulus were obtained using a load-controlled UMIS nanoindenter equipped with a Berkovich diamond indenter with a tip radius of approximately $150 \mathrm{~nm}$. An optimum load of $12 \mathrm{mN}$ was selected to avoid substrate effects and obtain load independent mechanical properties. A minimum of 30 indents for each film were used to evaluate the average and standard deviation of the contact hardness $(H)$ and elastic modulus $(E)$ using the Oliver and Pharr method [29].

\section{Results}




\subsection{Composition}

The as deposited monolithic film composition was determined to $\mathrm{Zr}_{0.43} \mathrm{Al}_{0.57} \mathrm{~N}$ and $\operatorname{TiN}( \pm 1.5$ at. \%) with nitrogen to metal ratio of $1 \pm 0.05$. Oxygen and carbon impurities account for less than 0.2 at. $\%$ while the Ar content is 0.8 at. $\%$ in the films. The $\mathrm{Al}$ content in the $\mathrm{Zr}-\mathrm{Al}-\mathrm{N}$ film is lower than that of the target by about 3 at. \%, which stems from the preferential re-sputtering of $\mathrm{Al}$ at the growth front $[14,30]$. The individual layer composition in the multilayers was estimated to be comparable to their monolithic counterparts based on the APT measurements.

\subsection{XRD analysis}

Figure 1 shows $\mathrm{x}$-ray diffractograms of the $\mathrm{Zr}_{0.43} \mathrm{Al}_{0.57} \mathrm{~N}$ film, multilayers with $l_{\text {ZraIN }}=2,10,15$ and $30 \mathrm{~nm}$ grown at $\mathrm{T}_{\mathrm{s}}=700{ }^{\circ} \mathrm{C}$, and the multilayer $l_{\text {zralN }}=15 \mathrm{~nm}$ grown at $\mathrm{T}_{\mathrm{s}}=900{ }^{\circ} \mathrm{C}$ in its as-deposited and annealed states. The TiN 002 reflection is overlapping with $\mathrm{MgO} 002$ due to their similar lattice constants. The monolithic film shows only one weak and broad diffraction signal around $32.4^{\circ}$. The peak is interpreted as $w$-AlN 0002 , shifted to a lower diffraction angle than pure $w$ - AlN due to the presence of $\mathrm{Zr}$ in the $w$-AlN lattice. The peak broadening is a result of a short coherent scattering length, which reflects the nanocrystalline nature of the film. The short period multilayers $l_{\text {ZrAIN }}=2$ and $5 \mathrm{~nm}$ exhibit finite thickness fringes around the TiN 002 reflection indicative of a superlattice nature of these multilayers. The diffraction signal originating entirely from 002 cubic reflections indicates epitaxial growth of these short period multilayers.

The appearance of a TiN 111 reflection in $l_{\text {ZraIN }}=10,15$, and $30 \mathrm{~nm}$ (hereafter, referred to as long period multilayer) suggests termination of epitaxial growth. The absence of a $c-\mathrm{ZrN}$ peak and the appearance of a broad diffraction peak around $32.4^{\circ}$ suggest that the $\mathrm{Zr}_{0.43} \mathrm{Al}_{0.57} \mathrm{~N}$ layers in the long period multilayers are structurally similar to the monolithic film. 
The multilayer, $l_{\text {ZrAIN }}=15 \mathrm{~nm}$ deposited at $\mathrm{T}_{\mathrm{s}}=900{ }^{\circ} \mathrm{C}$ shows a different diffraction pattern with a broad peak centered in between the positions of $c-\mathrm{ZrN} 002$ and $w$-AlN 0002. The suppression of the $c$-TiN 111 peak indicates that higher growth temperature promotes epitaxial growth in spite of higher layer thickness. Upon annealing at $1150{ }^{\circ} \mathrm{C}$, two broad peaks at $2 \theta \sim 35.77^{\circ}$ and $39.65^{\circ}$ appeared. The phase identification in these films was ambiguous due to broadness and overlap of several diffraction peaks, and will be discussed further combined with TEM results.

\subsection{Microstructure of as deposited films}

\subsubsection{Monolithic $\mathrm{Zr}_{0.43} \mathrm{Al}_{0.57} \mathrm{~N}$ with incoherent interfaces}

Figure 2 shows XTEM images and SAED of the $\mathrm{Zr}_{0.43} \mathrm{Al}_{0.57} \mathrm{~N}$ monolithic film. The HAADFSTEM image shows contrast variation with a wavelength of $\sim 3 \mathrm{~nm}$, attributed to the formation of $\mathrm{ZrN}$ and $\mathrm{AlN}$ domains during growth. The film displays weak columnar contrast in the bright field TEM image (Fig. 2a). The SAED pattern shows only diffraction signal from a wurtzite structure with 0002 preferential orientation in the growth direction. The lattice parameters determined from SAED pattern, $\mathrm{a} \approx 3.66 \AA$ and $\mathrm{c} \approx 5.41 \AA$, are larger than the equilibrium lattice parameter of $w$-AlN [31], indicating that segregated AlN-rich domains contain $\mathrm{Zr}$, in agreement with XRD results. The lattice resolved image in Fig. $2 \mathrm{~d}$ reveals preferentially oriented wurtzite crystallites with distorted regions occur every 2-3 nm.

These observations suggest that the segregated $\mathrm{Zr}_{0.43} \mathrm{Al}_{0.57} \mathrm{~N}$, assume a nanoscale $3 \mathrm{D}$ composite structure with incoherent interfaces, where the AlN-rich regions assume a wurtzite structure, while the ZrN-rich domains are distorted and gives no diffraction signal in XRD or SAED. Despite the distorted $\mathrm{ZrN}$-rich domains the nanocomposite film shows crystallographic texture as the surface energy minimization of $w$-AlN favors $<0002>$ orientation in the growth direction.

\subsubsection{Short period multilayers and coherent interfaces}


In the short period multilayers $l_{\text {ZrAlN }} 2$ and $5 \mathrm{~nm}$ well defined interfaces between TiN and $\mathrm{Zr}_{0.43} \mathrm{Al}_{0.57} \mathrm{~N}$ layers are observed in TEM (Fig. 3). STEM images show bright and dark modulations within the $\mathrm{Zr}_{0.43} \mathrm{Al}_{0.57} \mathrm{~N}$ layer that correspond to vertical aligned $\mathrm{ZrN}$ - and $\mathrm{AlN}$ rich domains with a wavelength of $\sim 2 \mathrm{~nm}$. The SAED patterns only contain cubic diffraction spots and no wurtzite phase is observed. The lattice resolved images shows cube-to-cube epitaxy between $\mathrm{TiN}$ and $\mathrm{Zr}_{0.43} \mathrm{Al}_{0.57} \mathrm{~N}$ layers. It means that the $\mathrm{AlN}$ - rich domains assume a metastable cubic lattice, forming coherent interfaces with both $\mathrm{TiN}$ and $\mathrm{ZrN}$, which leads to a self-alignment of the segregated domains.

The overall epitaxial growth of TiN and ZrAlN layers is also evident from the continuous columnar contrast of BF-TEM images (Fig 3c and f), more clearly seen for the multilayer of $l_{\text {ZrAIN }}=2 \mathrm{~nm}$, and from the inheritance of interlayer roughness in the growth direction in APT reconstructed tip of $l_{\text {ZralN }}=5 \mathrm{~nm}$ multilayers shown in Fig. $3 \mathrm{~g}$.

To understand the compositional effects of the metastable $c$-AlN phase formation, chemical intermixing between the layers is evaluated by the APT 1D-concentration profiles in the multilayer of $l_{\text {ZrAIN }}=5 \mathrm{~nm}$ as shown in Fig. $3 \mathrm{~h}$. An average interface width of $\sim 4 \mathrm{~nm}$ is estimated between TiN and $\mathrm{Zr}_{0.43} \mathrm{Al}_{0.57} \mathrm{~N}$ layers. This means that the actual thickness of the $\mathrm{Zr}_{0.43} \mathrm{Al}_{0.57} \mathrm{~N}$ layer shrinks to $\sim 1 \mathrm{~nm}$, while the rest of the layer consists of Ti intermixed $\mathrm{ZrN}$ and AlN- rich domains. Thus, in the $l_{\text {ZrAlN }}=2 \mathrm{~nm}$ multilayer the $\mathrm{Zr}_{0.43} \mathrm{Al}_{0.57} \mathrm{~N}$ layers are fully intermixed with TiN. These observations suggest that the short period multilayers undergo significant compositional modification at these high growth temperatures, which needs to be considered for metastable phase formation.

\subsubsection{Influence of growth temperature on the interface structure in long period multilayers}

The films with $l_{\mathrm{ZrAlN}}=10,15$, and $30 \mathrm{~nm}$ show similar $\mathrm{x}$-ray diffractograms and thus the multilayer $l_{\text {ZralN }}=15 \mathrm{~nm}$ is selected to be representative of long period multilayers. It was 
grown at $\mathrm{T}_{\mathrm{s}}=700$ and $900{ }^{\circ} \mathrm{C}$ to probe the variation on the interface structure of AlN domains.

Fig. 4 shows XTEM images of the multilayers grown at $700{ }^{\circ} \mathrm{C}(\mathrm{a}-\mathrm{c})$, and $900{ }^{\circ} \mathrm{C}(\mathrm{d}-\mathrm{f})$, respectively. STEM images at low and high magnifications reveal randomly distributed $\mathrm{ZrN}$ and AlN- rich domains in the ZrAIN layers grown at $\mathrm{T}_{\mathrm{s}}=700{ }^{\circ} \mathrm{C}$. The HR-TEM micrograph (Fig. 4c) shows a nanocomposite structure of $w$-AlN-rich and c-ZrN- rich (heavily distorted structure) domains similar to what has been observed in the monolithic film (see Fig. 2a), suggesting that the "template" effect of TiN layers is lost for the long period multilayers $\left(l_{\text {ZrAIN }}\right.$ $>10 \mathrm{~nm}$ ). Similar to the monolithic films, the only diffraction signal from the ZrAlN layer arises from the $w$-AlN-rich domains, observed as a weak 0002 reflection in Fig. 4a. The ZrNrich domains are too small and distorted to result in an observable diffraction signal, whereas the $c$-TiN shows nearly continues diffraction ring. These observations suggests that the $w$-AlN forms incoherent interfaces with both $\mathrm{ZrN}$ and $c$-TiN.

In contrast, the multilayer grown at $900{ }^{\circ} \mathrm{C}$ shows a different interface structure. The STEM micrograph reveal a compositionally modulated microstructure with vertically aligned domains within the $\mathrm{Zr}_{0.43} \mathrm{Al}_{0.57} \mathrm{~N}$ layer (Fig. $4 \mathrm{~d}$ and e), similar to what are observed in the short period multilayers. However, the SAED pattern shows well confined diffraction pattern of wurtzite and cubic phases, indicating that the $w$-AlN-rich domains form semi-coherent interfaces to $c$ - $\mathrm{ZrN}$ and $c$-TiN. Fast Fourier transform (FFT) of the lattice resolved image in combination with SAED (more clearly shown for the annealed films, Fig. 6b) yielded a coherency relation of $(110)_{\mathrm{c}-\mathrm{TiN}} / /(110)_{\mathrm{c}-\mathrm{ZrN}} / /(10-10)_{\mathrm{w}-\mathrm{AlN}}$ and $[001]_{\mathrm{c}-\mathrm{TiN}} / /[001]_{\mathrm{c}-\mathrm{ZrN}} / /[0001]_{\mathrm{w}-\mathrm{AlN}}$, here after termed type I orientation. A substrate-film SAED pattern (not shown here) revealed $(100)_{\mathrm{MgO}} / /(100)_{\mathrm{c}-\mathrm{TiN}}$ and $[001]_{\mathrm{MgO}} / /[001]_{\mathrm{c}-\mathrm{TiN}}$. The lattice resolved image (Fig. 4f) confirms the lattice coherency between $c-\mathrm{ZrN}$ and $w$-AlN domains, and between $c$ - $\mathrm{ZrN}$ domains and the $c$-TiN layer. As a consequence, the segregated domains form a chemically modulated and 
self-aligned structure within the $\mathrm{Zr}_{0.43} \mathrm{Al}_{0.57} \mathrm{~N}$ layer during growth. The type $I$ interfaces causes an expansion of the $c-\mathrm{ZrN}$ lattice and a shrinkage of the c-axis of $w$-AlN along the growth direction. This explains the single broad XRD peak between the positions of $c-\mathrm{ZrN} 002$ and $w$-0002 (see Fig.1). The SAED pattern also shows an additional weak cubic 111 reflection indicating that the film has an additional orientation, which evolves completely after annealing and will be addressed in section 3.4.

APT was performed to unveil under pinning compositional effects behind the structural variation of $l_{\mathrm{ZrAlN}}=15 \mathrm{~nm}$ multilayers as a function of growth temperature. Fig. $4 \mathrm{~g}$ shows $2 \mathrm{D}$ plan view $\mathrm{Al}$ concentration maps from $1 \mathrm{~nm}$ thick virtual slices of $\mathrm{Zr}_{0.43} \mathrm{Al}_{0.57} \mathrm{~N}$ layers from the multilayers grown at 700 and $900{ }^{\circ} \mathrm{C}$. The maps confirm the formation of $\mathrm{ZrN}$ - and $\mathrm{AlN}$ - rich domains. The more pronounced segregation for $\mathrm{T}_{\mathrm{s}}=900{ }^{\circ} \mathrm{C}$ is evident by a larger domain size and a higher frequency of AlN-rich domains. The average compositions ratios evaluated by proximity histogram are $\mathrm{Al}: \mathrm{Zr}=0.9$ for the $w$-AlN- rich domains and a $\mathrm{Zr}: \mathrm{Al}=0.7$ for the $c$ - $\mathrm{ZrN}$ rich domains. The values indicate that the AlN-rich domains are relatively pure. The APT 1-D profiles in Fig. $4 \mathrm{~h}$ show interface widths of $\sim 4 \mathrm{~nm}$ in the multilayers grown at 700 and $900{ }^{\circ} \mathrm{C}$, comparable to the values for short period multilayers. This suggests that the interface width is established by the ion-induced atomic mixing at the interface of layered structure, thus the difference in growth temperature is too small to have an effect in this case. The interface topographical roughness analysis shows that the bottom interface is rougher compared to the top interface of the $\mathrm{Zr}_{0.43} \mathrm{Al}_{0.57} \mathrm{~N}$ layer in both multilayers, likely caused by a faceted columnar growth of TiN layer below the $\mathrm{Zr}_{0.43} \mathrm{Al}_{0.57} \mathrm{~N}$ layer.

\subsection{Interface chemistry and crystallography after annealing}

The STEM micrograph in Fig. 5a, displays the multilayer $l_{\text {ZraIN }}=15 \mathrm{~nm}, \mathrm{~T}_{\mathrm{s}}=900{ }^{\circ} \mathrm{C}$, after isothermal annealing at $1150{ }^{\circ} \mathrm{C}$ for 2 hours. The AlN domains (dark contrast) are confined by 
the immiscible phases $\mathrm{ZrN}$ (bright contrast) in the lateral direction and $\mathrm{TiN}$ in the growth direction. As a consequence, the lateral coalescence of AlN domains is restricted to only $\sim 20$ $\mathrm{nm}$. However, the $\mathrm{Zr}_{0.43} \mathrm{Al}_{0.57} \mathrm{~N}$ displays a decrease in the layer thickness from $15 \mathrm{~nm}$ in the as deposited state to $5 \mathrm{~nm}$ after annealing. A comparison of EDX line spectra of the as deposited and annealed film (Fig. 5b) shows that $\mathrm{ZrN}$ and TiN interdiffuse. However, due to higher volume fraction of the TiN layer, diffusion of $\mathrm{ZrN}$ is dominant, leaving behind $\mathrm{AlN}$ as the main constituent in the original $\mathrm{Zr}_{0.43} \mathrm{Al}_{0.57} \mathrm{~N}$ layer, which explains the decrease in layer thickness.

An APT reconstructed tip in Fig. 5c shows the formation of pure AlN (red) domains with intermittent $\mathrm{ZrN}$ (blue) domains. The reconstruction also shows a relatively higher amount of $\mathrm{ZrN}$ out diffusion at the bottom interface, compared to the top interface of the $\mathrm{Zr}_{0.43} \mathrm{Al}_{0.57} \mathrm{~N}$ layer. Proximity analysis (Fig. 5d), shows concentration gradient of $\mathrm{Zr}$ with an average value of $10 \pm 1$ and $16 \pm 1$ at. $\%$ that results in a compositions of $\operatorname{Ti}_{0.8} Z_{0.2} \mathrm{~N} \pm 1$ at. $\%$ and $\mathrm{Ti}_{0.68} \mathrm{Zr}_{0.32} \mathrm{~N} \pm 1$ at. $\%$, respectively for the top and bottom interface. The higher volume fraction of $\mathrm{ZrN}$ at the bottom interface is also visualized as iso-concentration surfaces in Fig. 5d. We attribute this to the higher topographical roughness of the bottom interface of $\mathrm{Zr}_{0.43} \mathrm{Al}_{0.57} \mathrm{~N}$ in the as deposited film. The faceted TiN surface provides shorter diffusion path to the columnar boundaries, which explain higher amount of $\mathrm{ZrN}$ in the bottom interface. Whereas the top interface of the $\mathrm{Zr}_{0.43} \mathrm{Al}_{0.57} \mathrm{~N}$ is relatively smooth in the as deposited film. In Fig. 6, SAED pattern of the annealed multilayer shows confined reflections of cubic and wurtzite phases similar to the as deposited multilayer (see Fig. 4d). This is an important result indicating that the semi-coherent interfaces of $w$-AlN are stable even after isothermal annealing. However, it is to be noted that the $c-111$ reflections, which appeared weakly in the as deposited films and more distinctly after annealing. HR-TEM combined with FFT of lattice resolved images, SAED pattern, and our previous plan-view image analysis [11] revealed two 
different coherency relations of $w$-AlN lattice; type- $I: c-\mathrm{ZrN}_{(110)[001]} / w-\mathrm{AlN}_{(10-10)[001]}$ (Fig. 6c) in the lateral direction, and type II: $c$-TiN ${ }_{(111)[1-10]} / w$-AlN ${ }_{(0001)[11-20]}$ in the growth direction (Fig. 6d). Figure $6 \mathrm{~b}$ provides the visualization of the crystallographic details of both these non-isostructural semi-coherent interfaces, where $c-110 / w-10-10$ (type 1 ) $c-111 / w-0001$ (type II) have the same in plane symmetry.

The as deposited multilayer (Fig. 4d) clearly contains type 1 interfaces. Type II interfaces may coexist as a faint $c-111$ reflection is observed, i.e., type II interfaces may be initiated during growth and grow in extent during annealing. The analysis also explains the XRD peaks of the annealed film, where the diffraction peak at $35.77^{\circ}$ is originated from domains with type II interfaces and the peak at $39.65^{\circ}$ is from domains with the type I interfaces.

\subsection{Predicted interface energies}

First-principles calculations were performed to access the interfacial energetics of TiN/AlN and $\mathrm{ZrN} / \mathrm{AlN}$ by using multilayer models shown in Figure 7 . The results presented in Fig. 8 shows that the relative energy difference is low between the iso-structural coherent interfaces with (100) and (111) orientations for both TiN/AlN and ZrN/AlN. In the cases of hetro structural coherent interfaces we infer the following. Type $I$ is favorable for $\mathrm{ZrN} / \mathrm{AlN}$ interfaces but not for TiN/AlN, type II interfaces are energetically the most favorable ones for both material systems. The calculations also reveal that semi-coherent $c-\mathrm{TMN} / w-\mathrm{AlN}$ structural archetypes have relatively higher thermodynamic stability compared to the isostructurally coherent interfaces, i.e. $c-\mathrm{TMN} / c-\mathrm{AlN}$. This underlines the experimental observation of a high thermal stability of the film, $l_{\text {zralN }}=15 \mathrm{~nm}$ when it contains semicoherent interfaces.

The common in-plane lattice parameter of the multilayer and the strain values calculated in

each layer are listed in table 1 . In case of TiN/AlN, the strain values are largest for type I, 
followed by isostructural interfaces and are smallest for type II interfaces. In case of $\mathrm{ZrN} / \mathrm{AlN}$, the largest strain is found for isostructural interfaces, decreases for type $I$ and is smallest for type II interfaces. It is to be noted that the higher thermodynamic stability of a type II interface is related to the lower misfit strain, combined with the fact that the AIN is in its stable wurtzite structure. Accordingly, the structural misfit and the bulk free energy can be suggested as a measure to predict the relative thermodynamic stabilities in chemically modulated structures containing different coherent interface structures.

\subsection{Mechanical properties}

Figure 9a shows the variation in $H$ and $E$ of the monolithic and multilayered films as a function of $\mathrm{Zr}_{0.43} \mathrm{Al}_{0.57} \mathrm{~N}$ layer thicknesses for $\mathrm{T}_{\mathrm{s}}=700{ }^{\circ} \mathrm{C}$. The trend for $E$ follows closely the rule-of-mixture of monolithic TiN with a value of $458 \pm 15 \mathrm{GPa}$ and $\mathrm{Zr}_{0.43} \mathrm{Al}_{0.57} \mathrm{~N}$ with a value of $220 \pm 7 \mathrm{GPa}$. The multilayers display a hardness enhancement up to the $\mathrm{Zr}_{0.43} \mathrm{Al}_{0.57} \mathrm{~N}$ layer thickness of $5 \mathrm{~nm}$ with a value of $35 \pm 2 \mathrm{GPa}$ (Fig. 9a), where the chemical segregated domains grow epitaxially with the TiN layer. In contrast, the long period multilayers, which consist of a nanocomposite structure, with incoherent $w$-AlN, display a monotonic hardness drop as a function of $\mathrm{Zr}_{0.43} \mathrm{Al}_{0.57} \mathrm{~N}$ layer thickness. On the other hand, the multilayers with $l_{\text {ZrAlN }}=15 \mathrm{~nm}$, and $\mathrm{T}_{\mathrm{s}}=900{ }^{\circ} \mathrm{C}$, where the $w$-AlN form semi-coherent interfaces shows a relatively higher hardness in spite of a longer layer period (Fig. 9 b). More importantly, these multilayers display a stable $H$ value of $34 \pm 1.5 \mathrm{GPa}$, after isothermal annealing at $1150{ }^{\circ} \mathrm{C}$. Note that this value is significantly higher than both the monolithic and multilayers of TiAlN and ZrAlN reported previously that consists of incoherent interfaces. We ascribe the superior hardness of the annealed films in the current study to the thermally stable semi-coherent interfaces between $w$-AlN and $c$-TiN, $\mathrm{ZrN}$.

\section{Discussion:}


The current study investigates the crystal and interface structure of TiN/ZrAlN multilayer. TiN attain a stable cubic structure, and grow epitaxially on $\mathrm{MgO}$ due to close match of lattice constants [32]. $\mathrm{Zr}_{0.43} \mathrm{Al}_{0.57} \mathrm{~N}$ on the other hand is immiscible and segregates into $\mathrm{ZrN}$ and $\mathrm{AlN}$ rich domains during the growth. The growth temperature in the current study corresponds to $\sim 0.4 \mathrm{Tm}$, which sets the average ad-atom diffusion lengths to a few $\mathrm{nm}$ for the deposition rates used in this study [33], resulting in formation of a nanoscale composite structure of $\mathrm{ZrN}$ and AlN.

In the $\mathrm{TiN} / \mathrm{Zr}_{0.43} \mathrm{Al}_{0.57} \mathrm{~N}$ multilayers, the segregated $\mathrm{AlN}$ - rich domains form coherent, semicoherent, and incoherent interfaces with $c$-TiN and $c-\mathrm{ZrN}$ depending on the growth temperature and ZrAlN layer thickness as summarized in Figure 10. Interestingly, the semicoherent $w$-AlN/c-TMN interfaces display relatively high thermal stability, with a stable hardness of $34 \pm 1.5 \mathrm{GPa}$ even after elevated temperature annealing at $1150{ }^{\circ} \mathrm{C}$. This is significantly higher compared to previously reported monolithic and multilayers of TiAlN and ZrAlN films with incoherent interfaces [34][35]. The mechanisms governing the different interface structures and their effects on thermal and mechanical stability are discussed here separately.

\subsection{Influence of $\mathrm{T}_{\mathrm{s}}$ and $\boldsymbol{l}_{\mathrm{ZrAIN}}$ on crystal and interface structure}

A substrate temperature of $700{ }^{\circ} \mathrm{C}$ enables segregation within the $\mathrm{Zr}_{0.43} \mathrm{Al}_{0.57} \mathrm{~N}$ layers, however the ad-atom mobility is insufficient at the growth front for complete segregation into pure binaries. This information is extracted from APT analysis of the long period multilayers, revealing that approximately 10 at $\% \mathrm{Zr}$ on the metallic sub lattice of AlN domains and $\sim 30$ at $\% \mathrm{Al}$ on the metallic sub lattice of $\mathrm{ZrN}$-rich domains. We suggest that this oceurs substitutionary on the metal sublattice since the $\mathrm{N}$-content is nearly stoichiometrie. The high 
solute amount in $\mathrm{ZrN}$ - rich domains leads to a distorted lattice, whereas the lower solute concentration in the AlN- rich domains enable it to forms its stable wurtzite structure (Fig. 2d). The resulting nanocomposite structure of $w$-AlN and distorted $\mathrm{ZrN}$ forms incoherent interfaces both in monolithic film and in long period multilayers.

The coherent interfaces between AlN-rich domains and $c$-TiN and $c$ - $\mathrm{ZrN}$ only form in the short period multilayers $\left(l_{\text {ZralN }} \leq 5 \mathrm{~nm}\right)$ which is a consequence of the formation of metastable cubic AlN- rich domains. The epitaxial stabilization of metastable $c$ - AlN, caused by the minimization of the interface energy is reported in a wide range of multilayers when the layer thickness is less than the critical value [19,36,37]. However, chemical intermixing at layer interfaces generally occurs in multilayers and for short period multilayers these chemical gradients constitute a significant portion of the multilayer, as revealed in the case $l_{\text {ZrAIN }} \leq 5 \mathrm{~nm}$ (Fig. 3h). This has a positive effect on the formation of $c$-AlN by reducing the structural misfit with the template TiN layer. Hence, we suggest that the metastable phase formation in the short period multilayers is a combined effect of chemical intermixing and epitaxial stabilization. Beyond the critical layer thickness i.e. $l_{\text {ZralN }}>10 \mathrm{~nm}$, the template effect is lost and AlN domains assume its stable wurtzite structure with incoherent interfaces to the distorted $\mathrm{ZrN}$ domains similar to the monolithic films, which leads to termination of epitaxial growth between the layers of ZrAlN and TiN.

The transformation from incoherent to semi-coherent interfaces between $w$-AlN and the cubic phases ( $\mathrm{TiN}$ and $\mathrm{ZrN}$ ) in the long period multilayers was obtained by tuning the growth temperature. First of all, at higher deposition temperature $\left(\mathrm{T}_{\mathrm{s}} \sim 900{ }^{\circ} \mathrm{C}\right)$, the enhanced adatom mobility causes more pronounced segregation within the $\mathrm{Zr}_{0.43} \mathrm{Al}_{0.57} \mathrm{~N}$ layers (Fig. $4 \mathrm{~g}$ ). This reduces the solute induced distortion in $\mathrm{ZrN}$ - rich domains at $900{ }^{\circ} \mathrm{C}$, leading to a cubic structure. The interface energy minimisation between the nanoscale segregated domains of $c$ $\mathrm{ZrN}$ and $w$-AlN provide the thermodynamic driving force for the semi-coherent interface 
formation. Secondly, the higher adatom mobility provides the necessary atomic rearrangement on the growth front for the formation of semi-coherent interfaces between $c$ ZrN (110) and $w$-AlN (10-10). These two surfaces have similar in plane symmetry (Fig. 6b). The importance of the material selection to promote semi-coherent interfaces with $w$-AlN is noticeable here. For example, studies on TiAlN/MgO (001) performed under similar growth conditions as used in this study showed segregated domains of $c$-TiN and w-AIN with incoherent interfaces [38]. Our calculations also confirm higher thermodynamic stability of coherent interfaces between $w$-AlN (10-10) and $c$ - ZrN (110) compared to $c$-TiN (110), as the later one generates relatively higher misfit strain. Finally, this semi-coherency relationship leads (0001) and (002), as the growth front atomic planes for $w$-AlN and $c$ - $\mathrm{ZrN}$ consecutively, which keeps the semi-coherent growth front with a reduced surface energy. Subsequently, the nanoscale semi-coherent domains of $c-\mathrm{ZrN} / w$-AlN grow simultaneously by adapting a coupled growth similar to what has been observed for directionally solidified eutectic systems for a wide range of materials [39]. The result is a chemically and structurally modulated structure (Fig. 4e) with non-isostructural semi-coherent interfaces between $c$ - $\mathrm{ZrN}$ and $w$-AlN inherited across the TiN layer for the whole film. This results in type 1 interfaces with a misfit strain of $7 \%$. To reduce the strain energy further, a competitive type II interface is initiated, where the $c$-TiN (111) grows epitaxial to $w$-AlN (0001). The $\mathrm{MgO}(001)$ surface promotes type $I$, interfaces and the evolution of type $I I$ interfaces is attributed to a higher thermodynamic stability with a lower misfit strain, which is also shown in the calculations.

\subsection{Thermal stability of semi-coherent interfaces}

The structural misfit across semi-coherent interfaces generates strain energy, which increases with the domain size. At elevated temperature, coalescence of $w$-AlN results in larger domains and higher strain energy that could result in formation of incoherent interfaces to 
relive the strain energy. Therefore, the thermal stability is an important criteria that qualifies the semi-coherent structural archetype for the elevated temperature applications.

The competition between the strain and interface energy sets a critical domain size, above which the semi-coherency is likely to breakdown. In the case of TiAlN, the critical domain size is $\sim 15 \mathrm{~nm}$, where the coherent $c$-AlN transforms in to incoherent $w$-AlN [40]. This relatively low critical domain size is an effect of $c$-AlN being a metastable phase with an energy penalty of $0.18 \mathrm{eV} /$ atom with reference to its stable structure[41]. In contrast, for the $w$-AlN forming semi-coherent interfaces, the only driving force for the coherency breakdown stems from the strain energy. Therefore, the critical size of $w$-AlN domains to commence coherency breakdown is expected to be significantly larger and consequently results in higher thermal stability of the interfaces.

In the TiN/ZrAlN multilayers, the semi-coherency between $w$-AlN and cubic phases is retained after isothermal annealing at $1150{ }^{\circ} \mathrm{C}$ (Fig. 6). This is ascribed to: (i) relatively higher thermodynamic stability of type 1 orientation between $c-\operatorname{ZrN}(110) / w$-AlN (10-10) compared to iso-structural interfaces, (ii) the evolution of type II interfaces between $c$-TiN (111) / wAlN (0001) that lowers the strain energy of the film, (iii) the inter-diffusion between $\mathrm{ZrN}$ and TiN generating compositionally graded $c-\mathrm{Ti}(\mathrm{Zr}) \mathrm{N} / w$-AlN interfaces, which further reduces the misfit strain for type $I I$ interfaces as the in-plane lattice parameter misfit between $c$ $\operatorname{Ti}(\mathrm{Zr}) \mathrm{N}(111)$ and $w$-AlN (0001) becomes smaller, and (iv) limiting the coalescence of $w$-AlN domains to lower than the critical size, by confining them with the immiscible cubic phases.

The inter diffusion between $c$-TiN and $c-\mathrm{ZrN}$ in the current study suggests that they become miscible at elevated temperature, inspite they were predicted to have a positive enthalpy $(\Delta \mathrm{H}$ mix) of mixing [42] associated with a lattice mismatch of about 7\%. At the annealing temperature of $1150{ }^{\circ} \mathrm{C}$, it is likely that the entropy of mixing $\left(\Delta \mathrm{S}_{\text {mix }}\right)$ supersedes the enthalpy of mixing $\left(\Delta \mathrm{H}_{\text {mix }}\right)$, and thus provides the thermodynamic drive for the intermixing. This 
analysis is in line with the theoretical predictions [17] and previous observation by Rogström et al.[43].

\subsection{Influence of interface structure on mechanical properties}

Monolithic $\mathrm{Zr}_{0.43} \mathrm{Al}_{0.57} \mathrm{~N}$ shows significantly lower $H$ and $E$ values compared to the rest of the films. The lower shear resistance is likely caused by a coordinated shear displacement, similar to what has been shown for the nanostructured TMN thin films with incoherent interfaces $[44,45]$. The lower elastic modulus is a combined effect of higher volume fraction of $w$-AlN phase which has a higher compliance compared to $c$-TiN, AIN [15] and the distorted lattice of $\mathrm{ZrN}$ domains causing elastic softening similar to what has been observed for nanostructured metals [46].

The multilayers show a systematic variation of $H$ as a function of $\mathrm{Zr}_{0.43} \mathrm{Al}_{0.57} \mathrm{~N}$ layer thickness (Fig. 10a). For the short period multilayers consisting coherent interfaces between $c$-TiN/ $\mathrm{ZrN}$ and metastable $c$-AlN, the spatial fluctuation in elastic properties across the layers and within the $\mathrm{Zr}_{0.43} \mathrm{Al}_{0.57} \mathrm{~N}$ layer offer Koehler strengthening while the lattice misfit between the isostructural coherent interfaces result in coherency hardening. Both these strengthening mechanisms become more prominent for the multilayers with $5 \mathrm{~nm} l_{\mathrm{ZrAlN}}$, resulting in the highest $\mathrm{H}$ value of $35 \pm 2 \mathrm{GPa}$. The long period multilayers $\left(l_{\mathrm{ZrAlN}} \geq 10 \mathrm{~nm}\right)$ display a decrease in $\mathrm{H}$ as a function of $\mathrm{Zr}_{0.43} \mathrm{Al}_{0.57} \mathrm{~N}$ layer thickness.

Here, the incoherent interfaces between the nanoscale segregated domains of $w$-AlN and distorted $\mathrm{ZrN}$ domains offers relatively lower shear resistance similar to the monolithic $\mathrm{Zr}_{0.43} \mathrm{Al}_{0.57} \mathrm{~N}$ film. For the multilayers $l_{\text {ZralN }}=15 \mathrm{~nm}$, grown at $900{ }^{\circ} \mathrm{C}$, the evolution of semicoherent interfaces between $w$-AlN and $c$-TMN domains (Fig. 4d-f) make them resistant to the coordinated shear displacement which results in higher hardness (Fig. 9b).

More importantly, these multilayers show a stable and relatively high hardness $(34 \pm 1.5$ GPa) even after isothermal annealing at $1150{ }^{\circ} \mathrm{C}$. We suggest that the thermally stable semi- 
coherent interfaces between $w$-AlN and $c$-TMN domains offer both Koehler and coherency hardening similar to the isostructural interfaces between $c$-TiN and $c$-AlN. In addition, the non-isostructural semi-coherent interfaces provide an additional obstacles to dislocation glide due to the misorientation between the active glide planes (c-TiN $\{110\}$ and $w$-AlN $)[47,48]$. These findings show that semi-coherent $c$-TMN/w-AlN structural archetype achieves a high thermal stability when the interface crystallography and chemistry is tuned appropriately. Thermally stable semi-coherent interface structure between $c$-TMN/w-AlN leads to a higher and stable hardness during elevated temperature annealing.

\section{Conclusions}

$\mathrm{TiN} / \mathrm{Zr}_{0.43} \mathrm{Al}_{0.57} \mathrm{~N}$ multilayers consisting $c$-TiN, and segregated $c-\mathrm{ZrN}$, and $w / c-\mathrm{AlN}$, were grown using magnetron sputtering on $\mathrm{MgO}(001)$ substrates, where the interface structure was tuned between coherent, semi-coherent, and incoherent interfaces, by varying the multilayer design and the growth temperature.

AlN assume a metastable cubic structure characterized with limited thermal stability with $l_{\text {ZrAIN }} \leq 5 \mathrm{~nm}$. In contrast, AlN assumes the stable wurtzite structure in the multilayers with $l_{\text {ZrAIN }}>10 \mathrm{~nm}$ and the monolithic film. A growth temperature of $700{ }^{\circ} \mathrm{C}$ is inadequate to obtain complete segregation; instead the presence of $\mathrm{Al}$ in the $\mathrm{ZrN}$-rich domains causes a distorted $\mathrm{ZrN}$ lattice, which leads to incoherent interfaces between $\mathrm{ZrN}$ domains and the $w$-AlN lattice. Higher growth temperature $\left(\mathrm{T}_{\mathrm{s}}=900{ }^{\circ} \mathrm{C}\right)$ enables more pronounced segregation of $w$-AlN and $c$-ZrN during the growth, which results in domains having better crystal quality. The interface energy minimization of the nanoscale modulated structure leads to evolution of semi-coherent interfaces between $w$-AlN and cubic phases (TiN and $\mathrm{ZrN}$ ). Two types of coherency relations are found, $c-\mathrm{ZrN}_{(110)[001]} / w-\mathrm{AlN}_{(10-10)[001]}$ orientation is promoted by $\mathrm{MgO}(001)$ template effect and the $c-\mathrm{TiN}_{(111)[10-1]} / w-\mathrm{AlN}_{(0001)[11-20]}$ orientation is promoted by the higher thermodynamic stability, also revealed by the first principle calculations. 
Most importantly, the semi-coherency is retained after annealing at $1150{ }^{\circ} \mathrm{C}$ for $2 \mathrm{~h}$ which is attributed to higher thermodynamic stability of these interfaces structure and limited grain growth of $w$-AlN provided through confinement between $\mathrm{TiN}$ layers and $\mathrm{ZrN}$ domains. The semi-coherent interfaces offer both Koehler and coherency hardening mechanisms and thanks to thermally stable interfaces, the hardness retains to $\sim 34 \pm 1.5 \mathrm{GPa}$ after elevated temperature annealing.

In summary, we propose semi-coherent $w$-AlN structural archetype with appropriate interface structure as a promising approach to make films with high hardness and with improved thermal stability compared to films with isostructural $c$-TMN/c-AlN interfaces.

\section{Acknowledgements}

The European Union's Erasmus-Mundus graduate school in Material Science and Engineering (DocMASE), the Swedish Governmental Agency for Innovation Systems (Vinnova) through the VINNMER grant 2011-03464 are acknowledged for the financial support. The EU-funded project AME-Lab (European Regional Development Fund C/4EFRE-13/2009/Br) is acknowledged for the FIB/SEM use. The APT was financed by the DFG and the federal state government of Saarland (INST 256/298-1 FUGG).

\section{References:}

[1] A. Hörling, L. Hultman, M. Odén, J. Sjölén, L. Karlsson, Thermal stability of arc evaporated high aluminum-content $\mathrm{Ti}_{[1-\mathrm{x}]} \mathrm{Al}_{[\mathrm{x}]} \mathrm{N}$ thin films, J. Vac. Sci. Technol. A 20 (2002) 1815-1823.

[2] P.H. Mayrhofer, A. Hörling, L. Karlsson, J. Sjölén, T. Larsson, C. Mitterer, L. Hultman, Self-organized nanostructures in the Ti-Al-N system, Appl. Phys. Lett. 83 (2003) 2049.

[3] A. Knutsson, J. Ullbrand, L. Rogström, N. Norrby, L.J.S. Johnson, L. Hultman, J. Almer, M. P. Johansson Jöesaar, B. Jansson, M. Odén, Microstructure evolution during the isostructural decomposition of TiAlN-A combined in-situ small angle $\mathrm{x}$-ray scattering and phase field study, J. Appl. Phys. 113 (2013) 213518.

[4] R. Rachbauer, A. Blutmager, D. Holec, P.H. Mayrhofer, Effect of Hf on structure and age hardening of Ti-Al-N thin films, Surface \& Coatings Technology. 206 (2012) 2667-2672. 
[5] L. Chen, D. Holec, Y. Du, P.H. Mayrhofer, Influence of Zr on structure, mechanical and thermal properties of Ti-Al-N, Thin Solid Films. 519 (2011) 5503-5510.

[6] H. Lind, R. Forsén, B. Alling, N. Ghafoor, F. Tasnádi, M.P. Johansson, I.A. Abrikosov, M. Odén, Improving thermal stability of hard coating films via a concept of multicomponent alloying, Appl. Phys. Lett. 99 (2011) 091903.

[7] H. Lind, F. Tasnádi, I.A. Abrikosov, Systematic theoretical search for alloys with increased thermal stability for advanced hard coatings applications, New J. Phys. 15 (2013) 095010.

[8] A. Knutsson, M.P. Johansson, L. Karlsson, M. Odén, Thermally enhanced mechanical properties of arc evaporated $\mathrm{Ti}_{[0.34]} \mathrm{Al}_{[0.66]} \mathrm{N} / \mathrm{TiN}$ multilayer coatings, J. Appl. Phys. 108 (2010) 044312.

[9] R. Forsén, N. Ghafoor, M. Odén, Coherency strain engineered decomposition of unstable multilayer alloys for improved thermal stability, J. Appl. Phys. 114 (2013) 244303.

[10] Q. Xia, H. Xia, A.L. Ruoff, Pressure-induced rocksalt phase of aluminum nitride: A metastable structure at ambient condition, J. Appl. Phys. 73 (1993) 8198.

[11] N. Ghafoor, L.J.S. Johnson, D.O. Klenov, J. Demeulemeester, P. Desjardins, I. Petrov, L. Hultman, M. Odén, Nanolabyrinthine ZrAlN thin films by self-organization of interwoven single-crystal cubic and hexagonal phases, APL Mater. 1 (2013) 022105.

[12] A. Karimi, G. Allidi, R. Sanjines, Relative orientation of the constituents on the degree of crystallographic coherence in AlN/TiN superlattices, Surf. Coatings Technol. 201 (2006) 4062-4067.

[13] D. Rafaja, A. Poklad, V. Klemm, G. Schreiber, D. Heger, M. Š́ma, M. Dopita, Some consequences of the partial crystallographic coherence between nanocrystalline domains in Ti-Al-N and Ti-Al-Si-N coatings, Thin Solid Films. 514 (2006) 240-249.

[14] K. Yalamanchili, I.C. Schramm, E. Jiménez-Piqué, L. Rogström, F. Mücklich, M. Odén, N. Ghafoor, Tuning hardness and fracture resistance of $\mathrm{ZrN} / \mathrm{Zr}_{0.63} \mathrm{Al}_{0.37} \mathrm{~N}$ nanoscale multilayers by stress-induced transformation toughening, Acta Mater. 89 (2015) 22-31.

[15] A.J. Wang, S.L. Shang, Y. Du, Y. Kong, L.J. Zhang, L. Chen, D.D Zhao, Z.K. Liu, Structural and elastic properties of cubic and hexagonal TiN and AIN from firstprinciples calculations, Comput. Mater. Sci. 48 (2010) 705-709.

[16] M. Wen, H. Huang, K. Zhang, Q. Meng, X. Li, L. Kong, C. Hu, W. Zheng, The AlN layer thickness dependent coherent epitaxial growth, stress and hardness in NbN/AIN nanostructured multilayer films, Surface. Coatings Technol. 235 (2013) 367-375.

[17] H. Lind, R. Pilemalm, L. Rogström, F. Tasnadi, N. Ghafoor, R. Forsén, L.J.S. Johnson, M.P. Johansson-Jöesaar, M. Odén, I.A. Abrikosov, High temperature phase decomposition in TixZryAlzN, AIP Adv. 4 (2014) 127147.

[18] D. Holec, R. Rachbauer, L. Chen, L. Wang, D. Luef, P.H. Mayrhofer, Surface \& Coatings Technology Phase stability and alloy-related trends in $\mathrm{Ti}-\mathrm{Al}-\mathrm{N}, \mathrm{Zr}-\mathrm{Al}-$ $\mathrm{N}$ and $\mathrm{Hf}-\mathrm{Al}-\mathrm{N}$ systems from fi rst principles, Surf. Coat. Technol. 206 (2011) 1698-1704. 
[19] A. Madan, I.W. Kim, S.C. Cheng, P. Yashar, V.P. Dravid, S.A. Barnett, Stabilization of Cubic AlN in Epitaxial AlN - TiN Superlattices, Phy. Rev. Let. 78 (1997) 17431746.

[20] C. Stampfl, A.J. Freeman, Structure and stability of transition metal nitride interfaces from first-principles: AlN/VN, AlN/TiN, and VN/TiN, Appl. Surf. Sci. 258 (2012) $5638-5645$.

[21] M. Schlögl, B. Mayer, J. Paulitsch, P.H. Mayrhofer, Influence of CrN and AlN layer thicknesses on structure and mechanical properties of CrN/AIN superlattices, Thin Solid Films. 545 (2013) 375-379.

[22] N. Ghafoor, F. Eriksson, P.O.A.. Persson, L. Hultman, J. Birch, Effects of ion-assisted growth on the layer definition in Cr/Sc multilayers, Thin Solid Films. 516 (2008) 982990.

[23] M.K. Miller, K.F. Russell, Atom probe specimen preparation with a dual beam SEM/FIB miller, Ultramicroscopy. 107 (2007) 761-6.

[24] P. Bas, A. Bostel, B. Deconihout, D. Blavette, A general protocol for the reconstruction of 3D atom probe data, Appl. Surf. Sci. 87-88 (1995) 298-304.

[25] P.E. Blöchl, Projector augmented-wave method, Phys. Rev. B. 50 (1994) 1795317979.

[26] G. Kresse, Efficient iterative schemes for ab initio total-energy calculations using a plane-wave basis set, Phys. Rev. B. 54 (1996) 11169-11186.

[27] J.P. Perdew, K. Burke, M. Ernzerhof, Quantum Theory Group Tulane University, Generalized Gradient Approximation Made Simple, Phys. Rev. Lett. 77 (1996) 38653868.

[28] J.D. Pack, H.J. Monkhorst, special points for Brillouin-zone integrations, Phys. Rev. B. 16 (1977) 1748-1749.

[29] W.C. Oliver, G.M. Pharr, Measurement of hardness and elastic modulus by instrumented indentation: Advances in understanding and refinements to methodology, J. Mater. Res. 19 (2011) 3-20.

[30] B.M. Howe, E. Sammann, J.G. Wen, T. Spila, J.E. Greene, L. Hultman, et al., Realtime control of AlN incorporation in epitaxial $\mathrm{Hf}_{1-\mathrm{x}} \mathrm{Al}_{\mathrm{x}} \mathrm{N}$ using high-flux, low-energy $(10-40 \mathrm{eV})$ ion bombardment during reactive magnetron sputter deposition from a $\mathrm{Hf}_{0.7} \mathrm{Al}_{0.3}$ alloy target, Acta Mater. 59 (2011) 421-428.

[31] PDF-card No. 01-073-7288. JCPDS- International centre for diffraction data, 1998.

[32] L. Hultman, U. Helmersson, S. A. Barnett, J.-E. Sundgren, J.E. Greene, Low-energy ion irradiation during film growth for reducing defect densities in epitaxial TiN(100) films deposited by reactive-magnetron sputtering, J. Appl. Phys. 61 (1987) 552.

[33] D.G. Sangiovanni, D. Edström, L. Hultman, I. Petrov, J.E. Greene, V. Chirita, Ti adatom diffusion on $\mathrm{TiN}(001)$ : $\mathrm{Ab}$ initio and classical molecular dynamics simulations, Surf. Sci. 627 (2014) 34-41.

[34] A. Knutsson, M.P. Johansson, L. Karlsson, M. Odén, Thermally enhanced mechanical properties of arc evaporated $\mathrm{Ti}_{0.34} \mathrm{Al}_{0.66} \mathrm{~N} / \mathrm{TiN}$ multilayer coatings, J. Appl. Phys. 108 
(2010) 0-7.

[35] L. Rogström, L.J.S. Johnson, M.P. Johansson, M. Ahlgren, L. Hultman, M. Odén, Thermal stability and mechanical properties of arc evaporated $\mathrm{ZrN} / \mathrm{ZrAlN}$ multilayers, Thin Solid Films. 519 (2010) 694-699.

[36] H. Söderberg, M. Odén, T. Larsson, L. Hultman, J.M. Molina-Aldareguia, Epitaxial stabilization of cubic-SiN ${ }_{[x]}$ in TiNSiN ${ }_{[x]}$ multilayers, Appl. Phys. Lett. 88 (2006) 191902.

[37] V. Chawla, D. Holec, P.H. Mayrhofer, Stabilization criteria for cubic AlN in TiN/AIN and CrN/AlN bi-layer systems, J. Phys. D. Appl. Phys. 46 (2013) 045305.

[38] F. Adibi, I. Petrov, L. Hultman, U. Wahlström, T. Shimizu, D. McIntyre, J.E. Greene, J.E. Sundgren, Defect structure and phase transitions in epitaxial metastable cubic Ti0.5A10.5N alloys grown on $\mathrm{MgO}(001)$ by ultra-high-vacuum magnetron sputter deposition, J. Appl. Phys. 69 (1991) 6437-6450.

[39] J. Llorca, V.M. Orera, Directionally solidified eutectic ceramic oxides, Prog. Mater. Sci. 51 (2006) 711-809.

[40] N. Norrby, L. Rogström, M.P. Johansson-Jõesaar, N. Schell, M. Odén, In situ X-ray scattering study of the cubic to hexagonal transformation of $\mathrm{AlN}$ in $\mathrm{Ti}_{1_{-} \mathrm{x}} \mathrm{Al} \mathrm{x}_{\mathrm{x}} \mathrm{N}$, Acta Mater. 73 (2014) 205-214.

[41] V. Chawla, D. Holec, P.H. Mayrhofer, The effect of interlayer composition and thickness on the stabilization of cubic AlN in AlN / Ti - Al - N superlattices, Thin Solid Films. 565 (2014) 94-100.

[42] G. Abadias, V.I. Ivashchenko, L. Belliard, P. Djemia, Structure, phase stability and elastic properties in the $\mathrm{Ti}_{1-\mathrm{x}} \mathrm{Zr}_{\mathrm{x}} \mathrm{N}$ thin-film system: Experimental and computational studies, Acta Mater. 60 (2012) 5601-5614.

[43] L. Rogström, N. Ghafoor, M. Ahlgren, M. Odén, Auto-organizing ZrAlN/ZrAlTiN/TiN multilayers, Thin Solid Films. 520 (2012) 6451-6454.

[44] Z.B. Qi, P. Sun, F.P. Zhu, Z.C. Wang, D.L. Peng, C.H. Wu, The inverse Hall-Petch effect in nanocrystalline ZrN coatings, Surf. Coatings Technol. 205 (2011) 3692-3697.

[45] K. Yalamanchili, R. Forsén, E. Jiménez-Piqué, M.P. Johansson Jöesaar, J.J. Roa, N. Ghafoor, M. Odén, Structure, deformation and fracture of arc evaporated $\mathrm{Zr}-\mathrm{Si}-\mathrm{N}$ hard films, Surf. Coatings Technol. 258 (2014) 1100-1107.

[46] P. Sharma, S. Ganti, On the grain-size-dependent elastic modulus of nanocrystalline materials with and without grain-boundary sliding, J. Mater. Res. 18 (2011) 18231826.

[47] M. Odén, Characterization of the induced plastic zone in a single crystal TiN (001) film by nanoindentation and transmission electron microscopy, J. Mater. Res. 12 (1997) 2134-2142.

[48] S.R. Jian, Y.C. Tseng, I.J. Teng, J.Y. Juang, Dislocation Energetics and Pop-Ins in AlN Thin Films by Berkovich Nanoindentation, Materials. 6 (2013) 4259-4267. 
